\title{
Current and Emerging Treatments for Painful Diabetic Neuropathy
}

\author{
Cheng HT* \\ Headache and Neuropathic Pain Unit, Department of Neurology, Massachusetts General Hospital/ Harvard Medical School, USA
}

*Correspondence to: Hsinlin Thomas Cheng, Headache and Neuropathic Pain Unit, Department of Neurology, Massachusetts General Hospital/ Harvard Medical School, USA; E-mail: htcheng@mgh.harvard.edu

Received: March 26, 2018; Accepted: April 04, 2018; Published: April 06, 2018;

\section{Introduction}

Diabetes affects more than 30 million people in the United States with type 2 diabetes accounting for $90-95 \%$ of cases (www.diabetes. org). Annual medical expense and disease-related societal burden from diabetes cost more than $\$ 245$ billion. Most of the diabetic -related disabilities are from chronic diabetic complications in the cardiovascular, renal, retinal, and nervous systems. Among these, diabetic polyneuropathy occurs in approximately $60 \%$ of all diabetic patients $[1,2]$. Diabetic polyneuropathy causes significant public health burden, serving as the leading cause of diabetes-related hospital admissions and non-traumatic amputations $[1,3,4]$.

Patients with diabetic polyneuropathy frequently suffer from painful symptoms, termed as painful diabetic neuropathy (PDN) [2]. Clinically, PDN typically presents with length-dependent spontaneous pain with a combination of burning, tingling, electriclike, or achy sensations. It begins in the feet and extends proximally over time with bilateral and symmetric stocking distribution. Similar distal to proximal pattern of painful symptoms could develop at a later stage in the upper extremities. Patients with PDN also experience induced-pain, such as allodynia and hyperalgesia. Allodynia occurs when regularly innoxious stimuli, such as light touch, become painful, whereas hyperalgesia is increased nocuous sensitivity to painful stimuli, like pin prick. Despite the high morbidity of PDN $[5,6]$, the underlying molecular mechanisms of PDN are poorly understood [7]. Without targeting the key pathology that leads to the development of PDN, currently accepted medical approaches are only partially successful and are often ineffective $[5,8]$. Inadequate control of PDN has significantly reduced quality of life for patients with diabetes [5, $6,8]$. In addition to suffering from painful symptoms, patients with PDN frequently develop insomnia, depression and anxiety, decreased mobility, psychomotor impairment and loss of work $[5,6,8]$. Clearly, more mechanism-specific therapies are urgently needed to effectively manage this common and important health problem.

\section{Current treatment guidelines}

Over the last three decades, basic science and clinical studies have generated significant amount of evidenced-based data to establish treatment guidelines for PDN. The 2006 and 2010 guidelines from the European Federation of Neurological Societies task Force (EFNS) $[9,10]$ and the 2011 guidelines from the American Academy of
Neurology (AAN), the American Association of Neuromuscular and Electrodiagnostic Medicine, and American Academy of Physical Medicine and Rehabilitation [11] are the most thorough and up-todate guidelines on this topic. Several class drugs including $\alpha 2 \delta$ calcium channel antagonists (gabapentin, and pregabalin), anti-convulsants, tricyclic anti-depressants (TCAs), serotonin-norepinephrine reuptake inhibitors (SNRIs), opioids, and various other treatment modalities are discussed and recommended according to the quality of their supporting data. Each published clinical trial is classified according to its level of evidence, following guidelines such as the "AAN classification of recommendations" (www.AAN.com). Although there could be variations among these guidelines, trials deemed as class I are considered to have the highest quality of evidence with lowest risk of bias to support the application of the study drugs. The quality of evidence is decreased in high leveled classes; with class IV evidence has the highest bias potential and lowest supporting evidence for clinical use.

Level A treatments are strongly recommended with class I evidence or consistent findings from multiple studies of class II, III, or IV. They are recommended in clinical practice unless a clear and compelling rationale for an alternative approach is present. Level B treatments are with levels II, III, or IV evidence and findings are generally consistent. Generally, clinicians should follow this recommendation but should remain alert to new information and sensitive to patient preferences [12]. Level C, D, and U treatments do not have sufficient evidence to support their clinical practice.

The use of gabapentin, pregabalin, TCAs (such as amitriptyline), SNRIs (venlafaxine and duloxetine) are supported by EFNS with level A recommendation. In addition, controlled-release oxycodone is recommended by EFNS as effective with level A recommendation based on two class I studies. Tramadol alone or with acetaminophen were listed by the EFNS as level A effective treatments based on two class I studies. Level B recommendations from EFNS include Dextromethorphan (an agonist of N-methyl-D-aspartate receptor, 400 $\mathrm{mg} / \mathrm{d}$ ), Topical capsaicin $0.075 \%$ ointment that activates the transient receptor potential cation channel subfamily $\mathrm{V}$ member (TRPV) 1 , isosorbide dinitrate spray (a vasodilator), type A botulinum toxin (BTX-A, blocks acetylcholine release) and levodopa (a dopamine precursor) $[9,10]$. 
The guideline from AAN supports the use of pregabalin with level A recommendation. Gabapentin, sodium valproate (an anti-convulsant), venlafaxine, duloxetine, amitriptyline, dextromethorphan, morphine sulfate, tramadol, oxycodone, capsaicin $0.075 \%$ ointment, isosorbide dinitrate spray, electric stimulation and percutaneous nerve stimulation are presented as level B recommendations. Other anticonvulsants such as oxcarbazepine and lamotrigine; clonidine (an $\alpha 2$ adrenergic agonist), pentoxifylline (a xanthine derivative), magnetic field treatment, low-intensity laser therapy, and Reiki therapy are not recommended [11].

\section{Emerging treatments}

One of the most promising new gene therapies for PDN is a DNA-based therapy using a plasmid DNA that contains the human hepatocyte growth factor (HGF) gene (VM202). VM202 enhances local expression of HGF to promote microvasculature growth and regenerate peripheral nerves to improve symptoms of PDN. A phase 3 study showed that PDN patients receiving $8 \mathrm{mg}$ of VM202 injection per leg improved in all efficacy measures with $48.4 \%$ of the patients experienced at least a $50 \%$ reduction in mean pain score in the treated group compared with $17.6 \%$ in the placebo group after 3 months [13]. However, this analgesic effect was not statistically significant at 6 and 9 months. The study also demonstrated significant improvement in the brief pain inventory and the questionnaire portion of the Michigan Neuropathy Screening Instrument. Interestingly, the researchers noted that the largest reductions in pain were found among patients not on pregabalin or gabapentin. In addition, there were no significant adverse events attributable to VM202 and this treatment was deemed safe and well tolerated [13].

A network meta-analysis accumulated 25 randomized controlled trials for studying the effects of capsaicin $179 \mathrm{mg}$ cutaneous patch (capsaicin $8 \%$ patch) on PDN. It was concluded that capsaicin $8 \%$ patch was significantly more effective than placebo with $\geq 30 \%$ pain reduction in PDN patients. In addition, capsaicin patch was statistically more efficacious when compared with pregabalin and gabapentin. It had similar efficacy while being compared with duloxetine [14].

Nerve growth factor (NGF) has been established as an essential factor for the development of nociceptive nerves. It also mediates the development of mechanical allodynia in animal model of type 2 diabetes [15]. Clinical trials using NGF neutralizing antibodies, including tanezumab and fulnatumab, have been reported with positive results for treating PDN. In the study that examined the effects of tanezumab in PDN, test subjects received subcutaneous tanezumab $20 \mathrm{mg}$ or placebo on Day 1 and Week 8. Mean PDN pain reduction from baseline to Week 8 was greater with tanezumab vs placebo. However, differences in Patient's Global Assessment of DPN were not significant [16]

Fulranumab, a fully human monoclonal anti-NGF antibody was also tested for PDN. In a phase II, double-blind, placebo-controlled trial, patients with moderate to severe PDN were randomized to treatments with fulranumab $(1,3$, or $10 \mathrm{mg})$ or placebo administered subcutaneously every 4 weeks. Because of early study termination (clinical hold by the US Food and Drug Administration), only 77 of the planned 200 patients were enrolled. The primary endpoint, the mean reduction of average daily pain at week 12 compared with baseline, showed a positive dose-response relationship. The pair-wise comparison between the 10-mg group and placebo was significant. An exploratory responder analysis revealed that a greater proportion of patients in the $10-\mathrm{mg}$ group reported $\geq 30 \%$ reduction in the average pain intensity compared with placebo at week 12 . During the combined efficacy and safety extension phases, the top 3 treatmentemergent adverse events in the combined fulranumab group were arthralgia (11\%), peripheral edema (11\%), and diarrhea (9\%). No cases of joint replacement or death were reported [17]. Despite early study termination, fulranumab treatment resulted in dose-dependent efficacy and was generally well tolerated.

ARA 290 is a nonhematopoietic peptide designed from the structure of erythropoietin. In this trial, ARA 290 (4 mg) or placebo were self-administered subcutaneously daily for 28 days and the subjects followed for an additional months without further treatment. During the 56-day observation period, subjects with ARA 290 treatments had improvement in hemoglobin A1c (Hb A1c) and lipid profiles. Neuropathic pain from PDN improved significantly in the ARA 290 group. In addition, subjects with $>1$ standard deviation reduction in mean corneal nerve fiber density (CNFD) showed a significant improvement in CNFD compared with no change in the placebo group [18].

Botulinum toxins (BoNTs) are used for treating multiple painful conditions. However, BoNTs are not yet approved for treating PDN in the United States. Multiple small-scaled clinical trials have provided evidence to support the use of type A BoNT (BTX-A) injections for PDN. A meta-analysis selected and analyzed the data from a class I [19] and class II [20] studies to examine the efficacy of BTX-A on PDN [21]. Combining the two qualifying studies, there were a total of 58 patients receiving a sum of 76 treatments for PDN randomly allocated to placebo or BTX-A treatments. The injected areas were identical in each trial with a fixed protocol using a $3 \times 4$ grid that was equally spaced to demarcate the injection sites on the dorsum of each foot. The class 2 study used OnabotulinumA while the class 1 study used AbobotulinumtoxinA. It was concluded that there was an improvement of 1.96 visual analogue scale points following treatment with BTX-A [21]. The results were concluded as clinically significant improvement of "minimum change in pain." No serious adverse effects were reported in both trials. The meta-analysis evaluated the significance, low overall risk of bias, and almost no statistical heterogeneity support a correlation between Botox and improvement of pain scores for treating PDN [21]. However, further large scale controlled trials are needed to further establish the clinical efficacy and safety for this potential new indication for BTX-A.

\section{Future study strategies}

As reviewed in the current article, promising evidence support that several emerging treatments could be available for treating PDN in the near future. Other novel strategies are also under extensive study for developing new PDN treatments.

Animal studies have provided evidence that neurogenic inflammation in skin could be an important pathomechanisms for the 
development of PDN [22]. In a mouse model of type 2 diabetes, skin inflammatory cells (such as macrophages and Langerhans cells) could be activated by NGF signaling to target intraepidermal nerve fibers and be responsible for the development of pain behaviors. New evidence suggests that cytokine dysregulation could contribute to these skin inflammatory phenomena and suggest using immuno-modulatory therapies could be a novel treatment strategy for PDN [23].

Sodium channel $\mathrm{NaV}$ 1.7, $\mathrm{NaV} 1.8$, and $\mathrm{NaV} 1.9$ (encoded by SCN9A, SCN10A, and SCN11A respectively) are preferentially expressed in peripheral sensory neurons for nociception. Sodium channel Nav1.7 antagonists, including Xenon 402, CNV1014802, and PF-05089771, are being tested as new therapies for PDN [24]. Taken together, accumulating data from evidence-based studies shine light to the promising future of PDN management.

\section{References}

1. Feldman EL (2001) Diabetic neuropathy, in Principles and Practice of Endocrinology and Metabolism. Philadelphia, Lippincott Williams \& Wilkins, USA, pp. 1391-139.

2. Feldman EL (2003) Somatosensory neuropathy, in Ellenberg and Rifkin's Diabetes Mellitus. Pennsylvania, McGraw Hill, USA, pp: 771-788.

3. Feldman EL (1999) Diabetic neuropathy, in Current Review of Diabetes. Current Medicine 71-83.

4. Feldman EL (1999) Diabetic neuropathy, in Diabetes in the New Millennium. The Endocrinology and Diabetes Research Foundation of the University of Sydney: Sydney. pp: $387-402$.

5. Dworkin RH, Turk DC, Farrar JT, Haythornthwaite JA, Jensen MP, et al. (2005) Core outcome measures for chronic pain clinical trials: IMMPACT recommendations. Pain 113: 9-19. [Crossref]

6. Jensen MP, Dworkin RH, Gammaitoni AR, Olaleye DO, Oleka N, et al. (2006) Do pain qualities and spatial characteristics make independent contributions to interference with physical and emotional functioning? J Pain 7: 644-653. [Crossref]

7. Galer BS, Gianas A, Jensen MP (2000) Painful diabetic polyneuropathy: epidemiology, pain description, and quality of life. Diabetes Res Clin Pract 47: 123-128. [Crossref]

8. Dworkin RH, Jensen MP, Gammaitoni AR, Olaleye DO, Galer BS. (2007) Symptom profiles differ in patients with neuropathic versus non-neuropathic pain. $J$ Pain 8 : 118-126. [Crossref]

9. Attal N (2006) EFNS guidelines on pharmacological treatment of neuropathic pain. Eur J Neurol 13: 1153-1169. [Crossref]

10. Attal N, Cruccu G, Baron R, Haanpää M, Hansson P, et al. (2010) EFNS guidelines on the pharmacological treatment of neuropathic pain: 2010 revision. Eur J Neurol 17: 1113. [Crossref]

11. Bril V, England J, Franklin GM, Backonja M, Cohen J, et al. (2011) Evidencebased guideline: Treatment of painful diabetic neuropathy: report of the American Academy of Neurology, the American Association of Neuromuscular and Electrodiagnostic Medicine, and the American Academy of Physical Medicine and Rehabilitation. Neurology 76: 1758-1765. [Crossref]

12. Burns PB, Rohrich RJ, Chung KC (2011) The levels of evidence and their role in evidence-based medicine. Plast Reconstr Surg 128: 305-310. [Crossref]

13. Kessler JA, Smith AG, Cha BS, Choi SH, Wymer J, et al. (2015) Double-blind, placebo-controlled study of HGF gene therapy in diabetic neuropathy. Ann Clin Transl Neurol 2: 465-478. [Crossref]

14. van Nooten F, Treur M, Pantiri K, Stoker M, Charokopou M (2017) Capsaicin $8 \%$ Patch Versus Oral Neuropathic Pain Medications for the Treatment of Painful Diabetic Peripheral Neuropathy: A Systematic Literature Review and Network Meta-analysis. Clin Ther 39: 787-803 e18. [Crossref]

15. Cheng HT, Dauch JR, Hayes JM, Hong Y, Feldman EL (2009) Nerve growth factor mediates mechanical allodynia in a mouse model of type 2 diabetes. $J$ Neuropathol Exp Neurol 68: 1229-1243. [Crossref]

16. Bramson C, Herrmann DN, Carey W, Keller D, Brown MT et al. (2015) Exploring the role of tanezumab as a novel treatment for the relief of neuropathic pain. Pain Med 16: 1163-1176. [Crossref]

17. Wang H, Romano G, Frustaci ME, Bohidar N, Ma H, et al. (2014) Fulranumab for treatment of diabetic peripheral neuropathic pain: A randomized controlled trial. Neurology 83: 628-637. [Crossref]

18. Brines M, Dunne AN, van Velzen M, Proto PL, Ostenson CG et al. (2015) ARA 290, a nonerythropoietic peptide engineered from erythropoietin, improves metabolic control and neuropathic symptoms in patients with type 2 diabetes. Mol Med 20: 658-666. [Crossref]

19. Ghasemi M, Ansari M, Basiri K, Shaigannejad V (2014) The effects of intradermal botulinum toxin type a injections on pain symptoms of patients with diabetic neuropathy. J Res Med Sci 19: 106-111. [Crossref]

20. Yuan RY, Sheu JJ, Yu JM, Chen WT, Tseng IJ, et al. (2009) Botulinum toxin for diabetic neuropathic pain: a randomized double-blind crossover trial. Neurology 72: 1473-1478. [Crossref]

21. Lakhan SE, Velasco DN, Tepper D (2015) Botulinum Toxin-A for Painful Diabetic Neuropathy: A Meta-Analysis. Pain Med 16: 1773-1780. [Crossref]

22. Dauch JR, Bender DE, Luna-Wong LA, Hsieh W, Yanik BM, et al. (2013) Neurogenic factor-induced Langerhans cell activation in diabetic mice with mechanical allodynia. J Neuroinflammation 10: 64. [Crossref]

23. Zhang C, Ward J, Dauch JR, Tanzi RE, Cheng HT (2018) Cytokine-mediated inflammation mediates painful neuropathy from metabolic syndrome. PLoS One 13. [Crossref]

24. Bagal SK, Marron BE, Owen RM, Storer RI, Swain NA(2015) Voltage gated sodium channels as drug discovery targets. Channels (Austin) 9: 360-366. [Crossref]
Citation:

Cheng HT (2018) Current and Emerging Treatments for Painful Diabetic Neuropathy. J Clin Res Med Volume 1(2): 1-3 